\title{
Design of Feedforward Controller with Predicted Structural Velocity using Modal Expansion Method
}

\author{
Jeong-Mo Ku ${ }^{1}$, Min-Jung Sim ${ }^{1}$, Weui-Bong Jeong ${ }^{1}$, Chinsuk Hong ${ }^{2}$ \\ ${ }^{1}$ Department of Mechanical Engineering, Pusan National University \\ Jang-Jeon Dong, Busan, Korea \\ ustaryou@pusan.ac.kr; cldbzl5281@daum.net; wbjeong@pusan.ac.kr \\ ${ }^{2}$ Department of Mechanical Engineering, Ulsan College \\ Mu-Geo Dong, Ulsan, Korea \\ cshong@uc.ac.kr
}

\section{Extended Abstract}

When a controller is designed to minimize the kinetic energy with the feedforward strategy, the velocities of the entire structure should be measured for designing the controller[1]. Significant advantages can be obtained by implementation of the estimation process of the structural velocities from the measurement at a limited number of sensors. The advantages are denoted by the simpler architecture of the controller and the cost effectiveness owing to the sensor reduction. This paper is concerned with feedforward control to minimize the kinetic energy of the structure calculated by the velocity field estimated by modal expansion method based on the measured velocity at the limited number of sensors[2].

Minimization of the kinetic energy of the structure is used as a cost function for the controller design of feedforward control strategy. The kinetic energy of the structure can be in general calculated by integrating the entire velocity field of the structure. To evaluate the entire velocity field using the measured velocity at the sensor locations, the modal expansion method is incorporated[3]. First, the finite element model is established for the structure, and is secondly used to calculate the mode vectors and modal contributions at each node. Using the results of the finite element analysis, the modal expansion method is completely implemented defining a modal participation factor of disturbance to give the entire velocity field from the limited measurement. And the modal participation factor of control source can be calculated by using natural frequency and mode vector. Then velocity field of entire structure can be expressed as linear sum of modal participation factors to the disturbance and the control source. The cost function, the kinetic energy of the structure, is formulated in the quadratic form of the modal expansion method matrix and velocity of sensing point. As a result, the optimal control force is obtained ensuring the kinetic energy to be minimized[4]. It is confirmed that the performance of the controller is enhanced at the frequency of interested under various conditions from the case of direct measurement of the cost function at several points.

\section{References}

[1] C. C. Fuller, S. Elliott, and P. A. Nelson, Active control of vibration. Academic Press, 1996.

[2] B.-K. Jung, W.-B. Jeong, and J.-H. Kim, "Estimation of Vibration and Radiated Noise of a Compressor by using Modal Expansion Method," in Proceedings of the Korean Society for Noise and Vibration Engineering Conference, 2012: The Korean Society for Noise and Vibration Engineering.

[3] B. K. Jung and W. B. Jeong, "Mode selection of modal expansion method estimating vibration field of washing machine," Journal of Sound and Vibration, vol. 340, pp. 343-353, 2015.

[4] C. Hong, "Active control of resiliently-mounted flexible structures," Ph.D. dissertation, University of Southampton, 2005. 